\title{
Applications of In Vitro-In Vivo Correlations in Generic Drug Development: Case Studies
}

\author{
Paramjeet Kaur, ${ }^{1,3}$ Xiaojian Jiang, ${ }^{1}$ John Duan, ${ }^{2}$ and Ethan Stier ${ }^{1}$
}

Received 16 January 2015; accepted 30 March 2015; published online 22 April 2015

\begin{abstract}
In vitro-in vivo correlation (IVIVC) is a predictive mathematical model describing the relationship between an in vitro property and a relevant in vivo response. The main objective of an IVIVC is to serve as a surrogate for human bioequivalence (BE) studies, which may reduce the number of BE studies performed during the initial approval process as well as with certain scale-up and postapproval changes. The US Food and Drug Administration (FDA) published a regulatory guidance related to development, evaluation, and applications of IVIVC for extended-release (ER) oral dosage forms in September 1997. Despite the publication of this guidance, the deficiencies related to IVIVC are still identified by the Division of Bioequivalence in the process of Abbreviated New Drug Application (ANDA) review. Thus, the main objective of this article is to present the most commonly occurring deficiencies associated with IVIVCs via selected case studies from the ANDAs for oral ER drug products only. We searched internal FDA databases from January 1996 to December 2014 to identify the ANDAs for proposed generic oral ER drug products containing IVIVC. Only 14 ANDA submissions had IVIVC data, and most were not acceptable. Only one ANDA submission included adequate information related to IVIVC data enabling the completion of BE review within first review cycle. It is hoped that awareness of the deficiencies presented in our article would help the generic drug applicants to submit complete and appropriate information related to IVIVC data, ultimately, resulting in a more timely approval of ANDAs.
\end{abstract}

KEY WORDS: bioequivalence; extended-release drug products; generics; IVIVC; SUPAC.

\section{INTRODUCTION}

An in vitro-in vivo correlation (IVIVC) is a predictive mathematical model describing the relationship between an in vitro property of a dosage form and a relevant in vivo response. Generally, the in vitro property is the rate or extent of drug dissolution or release while the in vivo response is plasma drug concentration or amount of drug absorbed (1). The in vitro dissolution testing of dosage forms has been extensively used for quality control purposes, to determine stable release characteristics of the product over time, to ensure batch-to-batch similarity, and formulation discrimination, etc. However, these in vitro dissolution tests may or may not be predictive of the in vivo performance of the dosage form (1-3). Thus, a main objective of developing and evaluating an IVIVC is to establish the dissolution test as a surrogate for human bioequivalence (BE) studies, which may reduce the number of $\mathrm{BE}$ studies required, when certain preapproval and postapproval changes are made in formulation composition, manufacturing process, equipment, and site. The IVIVC can also be used to support and/or validate the use of dissolution method and set dissolution specifications $(1,4-8)$. In recent years, there has been considerable interest

\footnotetext{
${ }^{1}$ Division of Bioequivalence II, Office of Generic Drugs, US Food and Drug Administration, Silver Spring, Maryland 20993, USA.

${ }^{2}$ Office of New Drug Quality Assessment, Office of Pharmaceutical Sciences, US Food and Drug Administration, Silver Spring, Maryland 20993, USA.

${ }^{3}$ To whom correspondence should be addressed. (e-mail: Paramjeet.Kaur@fda.hhs.gov)
}

within the pharmaceutical industry, academia, and regulatory sectors in IVIVCs. There are many published studies related to development and validation of IVIVC for various drugs and dosage forms (oral immediate release and extendedrelease dosage forms, modified-release parenteral dosage forms, and transdermal drug delivery systems, etc.) (6,8-10). In the USA, the IVIVC has been used by both the innovator and generic drug companies in the regulatory submissions due to the abovementioned applications. Majority of these IVIVCs are filed for oral extended-release (ER) drug products (11). This article focuses on the IVIVCs for generic oral ER drug products only.

The generic drug approval process requires that abbreviated new drug applicants demonstrate both pharmaceutical equivalence and $\mathrm{BE}$ between the generic product and its corresponding reference listed drug (RLD) product (innovator or brand) to be approved for marketing (12). Demonstrating BE in vivo in humans is the most preferred method of ensuring the therapeutic equivalence of generic drug product to the RLD product (13). Generally, in in vivo BE studies, the plasma concentration profile of the generic drug product is compared to that of RLD product, and two products are considered to be bioequivalent if they show the same rate and extent of absorption (14). In the presence of an established IVIVC, in vivo performance can be predicted from in vitro dissolution testing. In September 1997, the US Food and Drug Administration (US FDA) developed IVIVC guidance for ER dosage forms to minimize the need for in vivo bioavailability and BE studies as part of the formulation design and optimization (1). Although the IVIVC guidance for ER dosage forms contains the recommendation regarding how to develop and validate an IVIVC model, the 
deficiencies related to IVIVC development and validation are still identified by the Division of Bioequivalence, Office of Generic Drugs during the review process. Thus, the main objective of this article is to present the most commonly occurring deficiencies associated with IVIVCs via selected case studies from ANDA applications for oral ER drug products only. We hope that this information will help generic drug applicants to submit complete and appropriate information related to IVIVC data, which, in turn, will make the ANDA approval less time-consuming.

\section{Applications of IVIVC in Generic Drug Development}

The FDA IVIVC Guidance for Extended Release Oral Dosage Forms (1997) defines four levels of correlation: level A, level B, level C, and multiple level C. Level A and multiple level C IVIVCs are most useful for regulatory purposes. The readers are encouraged to read the FDA IVIVC Guidance (1997) for details on levels of IVIVCs, development and validation of IVIVCs, applications of IVIVC, and procedure to set dissolution specifications in the presence and absence of an established IVIVC (1).

In brief, for generic drug products, a predictive IVIVC can be used to serve as a justification for waiver of in vivo $\mathrm{BE}$ studies during the initial approval process as well as with certain scale-up and postapproval changes (SUPAC) and setting of dissolution specifications.

As mentioned above, for a generic drug product to be approved, the ANDA applicant must demonstrate BE to the RLD product. Generally, for multiple strength products, the highest strength of innovator drug product is listed as the RLD in the Orange Book, with some exceptions, mainly due to safety reasons (14). Mostly, in vivo BE studies are recommended only for the strength listed as RLD, and other strengths of the modified-release drug products may be deemed bioequivalent to the corresponding strengths of the reference product, if (1) the other strengths are in the same dosage form and are proportionally similar in its active and inactive ingredients to the strength on which acceptable BE studies were conducted; (2) the drug release mechanism from all strengths of the formulations is the same; and (3) the dissolution profiles of all strengths are similar in multimedia $(14,15)$. In case all strengths of the product line are not proportionally similar $(14,15)$, in vivo BE study is required to demonstrate that non-proportionally formulated strength of the generic drug product is bioequivalent to its corresponding reference product. In the presence of an established IVIVC, the requirement for an in vivo BE study for not proportionally similar strengths of the generic drug product line may be waived, if all strengths are qualitatively the same, have the same release mechanism, have similar in vitro dissolution profiles, and are manufactured using the same type of equipment, and the same manufacturing process at the same site as the strength on which BE study is conducted. In scenarios, where in vivo $\mathrm{BE}$ study is recommended on the lower or higher strengths of the test products due to nonlinear pharmacokinetics, the waiver of in vivo BE study for these strengths in the presence of an established IVIVC is not applicable.

For specific SUPAC-related changes under which a validated IVIVC can be used to support waiver of in vivo
BE study for oral ER drug products, the readers are directed to FDA IVIVC Guidance and SUPAC-MR Guidance $(1,16)$.

For generic ER drug products, the process of setting dissolution specifications starts by obtaining the dissolution profiles of at least 12 individual units of the generic drug product batch/lot (bio-batch/lot) used in the pivotal BE studies. To set dissolution specifications, the sufficient number of sampling points should be selected to adequately characterize the dissolution profile. Once an IVIVC is established, specifications should be established in such a way that optimally, all the lots that have dissolution profiles within the upper and lower limits of the specifications are bioequivalent and less optimally (but still possible) bioequivalent to an appropriate reference batch (1).

\section{METHODS}

In the present study, internal FDA databases were searched from January 1996 to December 2014 to identify the ANDAs for proposed generic oral ER drug products containing IVIVCs. For the purpose of this study, we identified the number of submissions containing IVIVCs, uses of IVIVC to support generic drug development, and deficiencies related to IVIVCs in the ANDAs.

\section{RESULTS}

We identified that only 14 ANDA submissions had IVIVC data. The ANDA applicants submitted the level A or multiple level C IVIVC for a number of reasons as mentioned in Table I. Out of these 14 IVIVCs, 4 IVIVCs were submitted in support of postapproval changes, 9 IVIVCs were submitted in support of preapproval changes, and 1 IVIVC was used to guide the development of to-be-marketed formulation. The dissolution method used for the development of IVIVC for the investigated ER dosage form in these 14 ANDAs is summarized in Table I.

For these 14 applications containing IVIVC data, only 3 had acceptable IVIVC data and 1 of these 3 applications included adequate IVIVC-related information enabling the completion of $\mathrm{BE}$ review within first review cycle. The main reasons why the majority of the IVIVCs were not deemed adequate are presented in the case studies discussed below. The representative seven case studies are selected to provide overview of the major issues identified during the review of IVIVC data submitted in the ANDAs.

Case Study 1. In this case, the applicant submitted multiple level $\mathrm{C}$ IVIVC to support changes in dissolution specifications. Three different formulations having slow, medium, and fast release were developed to obtain in vitro dissolution and in vivo plasma concentration-time data. The in vivo study was carried out as a four-way crossover study using three formulations with different release rates and a reference formulation. To develop a multiple level C IVIVC, in vitro dissolution data at different time points was correlated with the peak plasma drug concentration $\left(\mathrm{C}_{\max }\right)$ and area under the plasma concentration-time curve (AUC) values. The developed regression equations were then used to set dissolution specifications. To ensure that final specifications would result in formulations bioequivalent to the reference formulation, $C_{\max }$ and AUC were back-calculated from the 
Table I. Summary of IVIVC Data Submitted in the ANDAs

\begin{tabular}{|c|c|c|c|c|c|}
\hline Purpose of IVIVC & Dosage form & $\begin{array}{l}\text { Drug substance } \\
\text { BCS } \\
\text { classification }\end{array}$ & $\begin{array}{l}\text { Dissolution apparatus and } \\
\text { medium }\end{array}$ & $\begin{array}{l}\text { Number } \\
\text { of ANDAs } \\
\text { with IVIVC }\end{array}$ & $\begin{array}{l}\text { Number of } \\
\text { acceptable } \\
\text { IVIVCs }\end{array}$ \\
\hline $\begin{array}{l}\text { Change in dissolution method } \\
\text { and specifications }\end{array}$ & ER tablets & Class I or III & $\begin{array}{l}\text { USP apparatus II (paddle), } \\
0.1 \mathrm{~N} \mathrm{HCl}\end{array}$ & 1 & 0 \\
\hline Level 3 site manufacturing change & & Class I & $\begin{array}{l}\text { USP apparatus I (basket), } \\
0.1 \mathrm{~N} \mathrm{HCl}\end{array}$ & 1 & 0 \\
\hline Waiver for lower strengths & & Class I & $\begin{array}{l}\text { USP apparatus I (basket) } \\
\text { phosphate buffer, pH } 6.8\end{array}$ & 1 & 1 \\
\hline Waiver for higher strengths & ER tablets comprised & Class II & USP apparatus II (paddle), & 2 & 0 \\
\hline To support dissolution method & $\begin{array}{l}\text { of modified-release } \\
\text { core coated with } \\
\text { enteric coat }\end{array}$ & Class II & $\begin{array}{l}0.01 \mathrm{~N} \mathrm{HCl} \text { followed by } \\
\text { phosphate buffer, pH } 6.8\end{array}$ & & \\
\hline $\begin{array}{l}\text { Batch-to-batch variation in the } \\
\text { particle size, coating weight, } \\
\text { process changes, test product } \\
\text { composition do not impact } \\
\text { the BE }\end{array}$ & $\begin{array}{l}\text { ER tablets comprised } \\
\text { of controlled-release } \\
\text { pellets }\end{array}$ & Class I & $\begin{array}{l}\text { USP apparatus II (paddle), } \\
\text { phosphate buffer, pH } 6.8\end{array}$ & 3 & 1 \\
\hline Change in dissolution specifications & ER capsules & Class II & $\begin{array}{l}\text { USP apparatus I (basket), } \\
\text { water }\end{array}$ & 1 & 1 \\
\hline Change in dissolution specifications & $\begin{array}{l}\text { ER capsules containing } \\
\text { beads or pellets }\end{array}$ & Class I & $\begin{array}{l}\text { USP apparatus II (paddle), } \\
\text { phosphate buffer pH } 6.5\end{array}$ & 1 & 0 \\
\hline $\begin{array}{l}\text { Challenge the results of a failed } \mathrm{BE} \\
\text { study }\end{array}$ & & Class I & $\begin{array}{l}\text { USP apparatus II (paddle), } \\
\text { phosphate buffer pH } 7.5\end{array}$ & 1 & 0 \\
\hline $\begin{array}{l}\text { Batch-to-batch variation in pellet } \\
\text { coating does not impact the } \mathrm{BE}\end{array}$ & & Class I & $\begin{array}{l}\text { USP apparatus I (basket), } \\
\text { simulated gastric fluid } \\
\text { followed by simulated } \\
\text { intestinal fluid }\end{array}$ & 1 & 0 \\
\hline Change in dissolution specifications & ER oral suspension & Class I or III & $\begin{array}{l}\text { USP apparatus II (paddle), } \\
\text { phosphate buffer, pH 7.4 }\end{array}$ & 1 & 0 \\
\hline $\begin{array}{l}\text { Exploratory to guide the } \\
\text { development of pivotal } \\
\text { formulation }\end{array}$ & $\begin{array}{l}\text { ER tablets containing } \\
\text { both IR and ER } \\
\text { components }\end{array}$ & Class I & $\begin{array}{l}\text { USP apparatus VII (reciprocating } \\
\text { holder) at } 30 \mathrm{dpm}, 50 \mathrm{~mL} \text { of } \\
\text { acidified water, } \mathrm{pH} 3.0\end{array}$ & 1 & Not applicable \\
\hline
\end{tabular}

IVIVC in vitro-in vivo correlation, BCS Biopharmaceutics Classification System, ANDA Abbreviated New Drug Application, ER extendedrelease, $I R$ immediate-release, $B E$ bioequivalence

final specification limits using regression equations. The results showed that the back-calculated $C_{\max }$ and AUCs fall within the bioequivalence acceptance criteria. The agency confirmed that back-calculated $C_{\max }$ and AUC determined using the proposed dissolution specifications are bioequivalent. After clarification of the rationale for selecting reference formulation, the agency accepted the IVIVC data and concurred with the proposed changes to the dissolution specifications.

Case Study 2. The applicant submitted an amendment containing IVIVC data to support proposed changes to dissolution method for its marketed drug product. The applicant developed a level A IVIVC using its marketed formulation and the reference product formulation. The applicant considered the reference product formulation as the second formulation for IVIVC development. The limited multimedia dissolution testing data under different $\mathrm{pH}$ conditions was submitted. Internal and external predictabilities were not evaluated. Since the IVIVC development is formulation specific, it is not appropriate to use different formulations, i.e., test and reference, each from a different manufacturer. Also, use of bioequivalent formulations, i.e., test and reference having same release rate are not acceptable for IVIVC development, as IVIVC should be developed with formulations having different release rates. Per FDA IVIVC Guidance, use of only one formulation may be considered for a level A IVIVC for formulations for which in vitro dissolution is independent of the dissolution test conditions (e.g., $\mathrm{pH}$, medium, agitation). However, in this case, the applicant did not provide the complete dissolution data to show that in vitro dissolution is independent of dissolution conditions. Accordingly, the agency requested the applicant to submit additional in vitro dissolution data under different conditions to show that in vitro dissolution is independent of dissolution conditions. The applicant was also requested to develop IVIVC using formulations with different release rates if dissolution is condition dependent. Estimation of internal and/or external predictability was recommended. In response to the agency's concerns related to IVIVC data, the applicant withdrew its amendment for proposed changes in dissolution method.

Case Study 3. The applicant proposed a level 2 change for a non-release controlling excipient. Per SUPAC-MR Guidance, an in vivo $\mathrm{BE}$ study is not required to support level 2 changes in a non-release controlling excipient (16). However, this level 2 change in non-release controlling excipient resulted in a change in the dissolution behavior. To support the change in dissolution specifications beyond a 
$25 \%$ range due to this level 2 change in non-release controlling excipient for reformulated product, the applicant submitted a level A IVIVC. The IVIVC was developed using the original test product formulation and the reference product formulation, which were shown to be bioequivalent in the BE studies. External and internal predictabilities were not evaluated. As mentioned above, per FDA IVIVC Guidance, use of only one formulation may be considered for a level A IVIVC correlation for formulations for which in vitro dissolution is independent of the dissolution test conditions. However, in this case, in vitro dissolution data showed that drug release from the test formulation is condition dependent. Also, as mentioned above in case 2, use of different formulations (test and reference), each from a different manufacturer to develop IVIVC, is not acceptable. The agency determined that the relationship between the in vivo dissolution and the in vitro dissolution was formulation dependent as the regression equations for the test and reference formulations were different. This data indicated that developed IVIVC model cannot be used to predict the performance of any future formulations that were not used in the IVIVC model development. Due to the abovementioned reasons, the applicant's IVIVC was deemed inadequate. Ultimately, the applicant conducted in vivo BE studies comparing its reformulated test product to the RLD product. The dissolution specifications were then recommended by the agency based on in vitro dissolution testing conducted on the bio-lot (reformulated test product) used in the new BE studies.

Case Study 4. To seek approval for marketing of its generic drug product, the applicant developed a test product formulation that was shown to be bioequivalent to the corresponding reference product in in vivo BE studies. However, the applicant proposed quantitative variation in almost all the inactive ingredients used in the manufacture of its test product from batch to batch depending on the manufacturing in-process control. To support its claim that proposed batch-to-batch variation in its test product composition does not impact the $\mathrm{BE}$, the applicant submitted IVIVC data from the summary basis of approval (SBOA) for the RLD product. Since IVIVC is formulation specific, the applicant was requested to establish IVIVC using its test formulations differing in release rates. In this case, the applicant reformulated its test product and conducted new BE studies on the reformulated test product to seek approval for marketing.

Case Study 5. In this case, the applicant submitted IVIVC data from the literature to support its claim that batch-to-batch variation in pellet coating does not impact the $\mathrm{BE}$ of the test product compared to the reference product. The agency does not accept the published literature IVIVC data in lieu of in vivo BE studies. Thus, the applicant was recommended to conduct its own IVIVC study using a minimum of two new test formulations that differed adequately in release rate from the original test formulation used in the acceptable BE studies. The applicant did not submit the IVIVC data as recommended by the agency and withdrew its submission for other reasons.

Case Study 6. The applicant proposed a level 3 manufacturing site change. To support this change, the applicant submitted a level A IVIVC developed using three formulations with different release rates (i.e., slow, medium, and fast). In vitro dissolution profiles and in vivo plasma concentration profiles were obtained for these three formulations. The percent of dose released in vivo as a function of time was determined by using deconvolution technique. The deconvolution method requires the use of a weighting function for the "body system," known as the unit impulse response (17). In vivo data of an IR formulation was used to generate unit impulse response. Both internal and external predictabilities were assessed. The agency evaluated the submitted IVIVC data and found it unacceptable due to the following reasons: (i) the PK parameters could not be accurately predicted using the established IVIVC. The fast- and slow-releasing formulations had similar dissolution profiles, despite the fact that these two formulations showed marked differences in $C_{\text {max }}$ and AUC, and (ii) internal and external predictabilities were not confirmed. In response to the agency's concerns related to IVIVC data, the firm conducted an in vivo BE study to support the level 3 site change.

Case Study 7. In this case, the applicant's to-be-marketed formulation did not meet the $\mathrm{BE}$ acceptance criteria in an in vivo $\mathrm{BE}$ study. To challenge the results of failed $\mathrm{BE}$ study, the applicant established a level A IVIVC using the lower and higher strengths of the to-be-marketed product line. The lower strength was used as fast-releasing formulation, and the higher strength was used as slow-releasing formulation. The internal and external predictabilities were assessed. The agency determined that in vitro dissolution profiles of the lower and higher strengths of the to-be-marketed product line are similar, and in vitro dissolution is dependent on the dissolution conditions. Thus, the applicant was requested to develop IVIVC using formulations with different release rates or alternatively conduct an in vivo BE study. The applicant conducted a new in vivo BE study, which met the BE limits.

Besides the major deficiencies discussed above in the case studies, the most commonly, the applicants did not submit the complete IVIVC report to the agency. Only the summary report was submitted. At the agency's request, the applicants subsequently provided the complete IVIVC data. The following is a list of recurring common deficiencies related to IVIVC data in the ANDA submissions:

1. Failure to submit full IVIVC report required for the agency to make assessment.

2. Employment of formulation with only one release rate for the establishment of IVIVC without evidence to show that the dissolution is condition independent.

3. Use of formulations from different manufacturers to establish IVIVC.

4. Biowaiver request justified only by literature IVIVC data, i.e., peer review articles.

5. Failure to adequately assess external and/or internal predictability, i.e., the IVIVC model did not accurately predict the plasma concentration profiles and pharmacokinetic parameters in the range of release rates tested.

The majority of deficiencies presented above are avoidable. It is hoped that many of the issues identified in the case studies and commonly recurring deficiencies will be avoided 
in the future, by conducting the IVIVC in accordance with the criteria set forth in IVIVC Guidance for ER products and by providing the complete and appropriate IVIVC-related information. The above information is provided to assist applicants who submit IVIVC data in support of generic drug applications to the Office of Generic Drugs to prepare highquality submission containing complete information needed to make assessment within first review cycle.

\section{CONCLUSIONS}

A validated IVIVC adds in vivo relevance to the in vitro dissolution, which presents the opportunities to avoid in vivo $\mathrm{BE}$ testing in humans. An IVIVC can also be used to support wider dissolution specifications, where justified. From the data collected over 18 years, it was observed that only 14 ANDA submissions had IVIVC data and most were not acceptable. Furthermore, our limited experience show that even in the case of acceptable IVIVCs, the IVIVC data provided in the ANDA submissions was not adequate in the first review cycle. This article has illustrated the various deficiencies associated with the IVIVC data submitted in ANDA submissions through case studies. It is hoped that awareness of deficiencies that result in deficient IVIVCs for generic oral ER drug products will help avoid unnecessary review cycles of IVIVCs submitted in ANDA applications, allowing their approval in a more timely fashion.

Conflict of Interest This article reflects the views of the authors and should not be construed to represent FDA's views or policies.

\section{REFERENCES}

1. U.S. Food and Drug Administration/Center for Drug Evaluation and Research. Guidance for industry: extended release oral dosage forms: development, evaluation, and application of in vitro/in vivo correlations. Rockville: Food and Drug Administration. 1997. http://www.fda.gov/downloads/Drugs/GuidanceComplianceRegulatoryInformation/Guidances/UCM070239.pdf. Accessed 15 Dec 2014.

2. Chakraborty A, Pandya K, Aggarwal D. Establishing prospective IVIVC for generic pharmaceuticals: methodologies assessment. Open Drug Deliv J. 2014;5:1-7.

3. Qureshi SA. In vitro-in vivo correlation (IVIVC) and determining drug concentrations in blood from dissolution testing-a simple and practical approach. Open Drug Deliv J. 2010;4:38-47.
4. Uppoor VR. Regulatory perspectives on in vitro (dissolution)/in vivo (bioavailability) correlations. J Control Release. 2001;72:127-32.

5. Roudier B, Davit BM, Beyssac E, Cardot JM. In vitro-in vivo correlation's dissolution limits setting. Pharm Res. 2014;31:252938. doi:10.1007/s11095-014-1349-8.

6. Farrell C, Hayes S. IVIVC for oral drug delivery: immediate release and extended release dosage forms. In: Chilukuri DM, Sunkara G, Young D, editors. Pharmaceutical product development: in vitro-in vivo correlation. New York: Informa Healthcare; 2007. p. 125-39.

7. Modi NB, Lam A, Lindemulder E, Wang B, Gupta SK. Application of in vitro-in vivo correlation (IVIVC) in setting formulation release specifications. Biopharm Drug Dispos. 2000;21:321-6.

8. Emami J. In vitro-in vivo correlation: from theory to applications. J Pharm Pharm Sci. 2006;9:169-89.

9. Zolnik BS, Burgess DJ. In vitro-in vivo correlation on parenteral dosage forms. In: Krishna R, Yu L, editors. Biopharmaceutics applications in drug development. New York: Springer; 2008. p. 336-58.

10. Chaturvedula A, Banga AK. In vitro-in vivo correlation: transdermal drug delivery systems. In: Chilukuri DM, Sunkara G, Young D, editors. Pharmaceutical product development: in vitro-in vivo correlation. New York: Informa Healthcare; 2007. p. 153-76.

11. Buskirk GAV, Shah V, Yacobi A, et al. PQRI. Workshop Report: Application of ivive in formulation development. Dissolution Technologies. 2008. http://www.dissolutiontech.com/DTresour/ 201405Articles/DT201405_A07.pdf. Accessed 20 Feb 2015.

12. Approved products with therapeutic equivalence evaluations. 34th edition. Washington, DC: US Department of Health and Human Services, Public Health Service, Food and Drug Administration, Center for Drug Evaluation and Research, Office of Medical Products and Tobacco, Office of Generic Drugs. 2014. http://www.fda.gov/downloads/Drugs/DevelopmentApprovalProcess/UCM071436.pdf. Accessed 15 Dec 2014.

13. Code of Federal Regulations, Title 21, Part 320.24 (b), Revision April 1, 2014.

14. U.S. Food and Drug Administration Center for Drug Evaluation and Research. Guidance for industry: Bioavailability and bioequivalence studies for orally administered drug products-general considerations. Rockville: Food and Drug Administration. 2003. http://www.fda.gov/downloads/Drugs/ Guidances/ucm070124.pdf. Accessed 15 Dec 2014.

15. Food and Drug Administration Center for Drug Evaluation and Research. Guidance for industry: bioequivalence studies with pharmacokinetic endpoints for drugs submitted under an ANDA. Rockville: Food and Drug Administration. 2013. http:// www.fda.gov/downloads/drugs/guidancecomplianceregulatoryinformation/guidances/ucm377465.pdf. Accessed 20 Feb 2015.

16. U.S. Food and Drug Administration Center for Drug Evaluation and Research. Guidance for industry: SUPAC-MR: modified release solid oral dosage forms scale-up and postapproval changes: chemistry, manufacturing, and controls; in vitro dissolution testing and in vivo bioequivalence documentation. Rockville: Food and Drug Administration. 1997. http://www.fda.gov/downloads/Drugs/ Guidances/UCM070640.pdf. Accessed 17 Dec 2014.

17. Rettig H, Mysicka J. IVIVC: methods and applications in modified-release product development. Dissolution Technologies. 2008. http://www.dissolutiontech.com/DTresour/200802Articles/ DT200802_A01.pdf. Accessed 20 Dec 2014. 